\title{
Assessment of the naphthalene sublimation technique for determination of convective heat transfer in fundamental and industrial applications
}

\author{
B. Niebles Atencio ${ }^{1}$ (D) $\cdot$ H. Jamshidi ${ }^{1} \cdot$ M. Liljemark ${ }^{2} \cdot$ H. Nilsson ${ }^{1} \cdot$ V. Chernoray ${ }^{1}$
}

Received: 21 April 2019 / Accepted: 3 December 2019 / Published online: 14 December 2019

(C) The Author(s) 2019

\begin{abstract}
The naphthalene sublimation technique is an experimental method for indirectly determining convective heat transfer. The technique is here assessed for two different configurations: the local heat transfer distribution for a circular air jet impinging normal to a flat surface, and the heat transfer occurring in the stator core of an electric generator model. The turbulent impinging jet is fully developed. Two Reynolds numbers based on the nozzle exit condition, 15000 and 23000, and two nozzle diameter distances from the jet exit to the surface, 6 and 8, are considered. For the generator turbulent internal flow with Reynolds number of 4100 is considered, based on the hydraulic diameter of stator ventilation ducts. Modern surface scanning methods and imprints of the naphthalene specimens were used for measuring the naphthalene sublimation rate. The impinging jet results are compared with experimental data found in the literature. Results from the generator model and numerical simulations are compared. For the impinging jet, the results show agreement with the already published experimental data sets. For the generator model, heat transfer results from experiments differ by around 13\% compared to numerical results if a scanning of the surface is used for measuring the naphthalene sublimation and around 5\% if weights are used for measuring the sublimation rate. Therefore, the results depend on the way the sublimation rate is quantified. From this study, it is possible to affirm that with advanced scanning procedures, the heat transfer can be resolved with very small naphthalene sublimation in cases of both fundamental and complex industrial applications such as electric generators.
\end{abstract}

\section{Nomenclature}

Sc $\quad$ Schmidt number.

$\mathrm{Pr} \quad$ Prandtl number.

$d \quad$ Inner diameter of the nozzle, $m$.

$d_{h} \quad$ Hydraulic diameter, $m$.

$U \quad$ Bulk velocity, $m / s$.

$\nu \quad$ Kinematic viscosity, $\mathrm{m}^{2} / \mathrm{s}$.

Re Reynolds number, $U d / \nu$.

$R e_{d h} \quad$ Reynolds number based on hydraulic diameter, $U d_{h} / \nu$.

$H \quad$ Nozzle exit to plate distance, $m$.

$Z \quad$ Normal coordinate, $m$.

$\frac{h_{m}}{h_{m}} \quad$ Local and average mass transfer coefficient, $\mathrm{m} / \mathrm{s}$.

B. Niebles Atencio

bercelay.niebles@chalmers.se

1 Department of Mechanics and Maritime Sciences, Chalmers University of Technology, Gothenburg, Sweden

2 RISE, Research Institute of Sweden, Measurement Science and Technology, Borås, Sweden $r \quad$ Radial coordinate, $m$.

$S h, \overline{S h} \quad$ Local and average Sherwood number.

$\delta Z \quad$ Local sublimation depth, $m$.

$\delta t \quad$ Time duration of the test, $s$.

$\delta m \quad$ Difference in weights of the specimen, $g$.

$A_{s} \quad$ Area of naphthalene surface, $m^{2}$.

$\dot{M}^{\prime} \quad$ Local naphthalene mass flux, $\mathrm{kg} / \mathrm{s}-\mathrm{m}^{2}$.

$\rho_{v, \infty} \quad$ Density of naphthalene in upstream air, $\mathrm{kg} / \mathrm{m}^{3}$.

$\rho_{v, w} \quad$ Density of naphthalene vapor, $\mathrm{kg} / \mathrm{m}^{3}$.

$\rho_{s} \quad$ Density of solid naphthalene, $\mathrm{kg} / \mathrm{m}^{3}$.

$D_{\text {naph }} \quad$ Diffusivity of naphthalene vapor in air, $\mathrm{m}^{2} / \mathrm{s}$.

$T \quad$ Temperature, $\mathrm{K}$.

$N u, \overline{N u} \quad$ Local and average Nusselt number.

$P_{\text {atm }} \quad$ Atmospheric pressure, $N / m^{2}$.

$n \quad$ Empirical constant.

$y^{+} \quad$ Dimensionless wall distance.

$k \quad$ Turbulent kinetic energy, $\mathrm{m}^{2} / \mathrm{s}^{2}$.

$\omega \quad$ Specific turbulent dissipation rate, $1 / s$.

$X \quad$ Data X-coordinate, $m$.

$Y \quad$ Data y-coordinate, $m$.

$\overline{N u_{N i}} \quad$ Normalized Nusselt number.

$\overline{N u_{i}} \quad$ Average Nusselt number in any row. 


$\begin{array}{ll}\overline{N u_{4 s}} & \begin{array}{l}\text { Average Nusselt number from the scanning } \\ \text { in row } 4 .\end{array} \\ \text { Acronyms } \\ \text { CFD } & \text { Computational Fluid Dynamics. } \\ \text { SST } & \text { Shear-stress transport. } \\ \text { RANS } & \text { Reynolds Average Navier-Stokes. } \\ \text { DS } & \text { Downstream side of the ventilation channel. } \\ \text { US } & \text { Upstream side of the ventilation channel. } \\ \text { CMM } & \text { Coordinate measurement machine. } \\ \text { SLS } & \text { Selective Laser Sintering. }\end{array}$

\section{Introduction}

Convective heat transfer coefficients are often determined by complex experiments involving advanced instruments and difficult procedures. These procedures are even more difficult when the local heat transfer distribution over an entire test section in a practical engineering model with a complex geometry is needed. An alternative way to indirectly obtain the convective heat transfer coefficients is to conduct mass transfer experiments, which have better accuracy and are usually easier to perform. The analogy between heat and mass transfer is then used to obtain the heat transfer. One of the most convenient mass transfer methods is the well-developed naphthalene sublimation technique. Naphthalene is favorable for mass transfer experiments because it possesses desirable properties such as low toxicity and a sublimation that occurs at room temperature. It also has good machining and casting properties. There are many advantages of using the naphthalene sublimation technique rather than doing direct heat transfer experiments. The naphthalene sublimation technique is particularly useful in complex flows and complex geometries with restrictions in the visual access. Large gradients in transfer rate are difficult to capture in heat transfer measurements, but the naphthalene sublimation technique can be used to determine them even at corners and edges. Mass transfer boundary conditions analogous to isothermal and adiabatic walls in convective heat transfer can be easily imposed in this method. The surface that is coated or made of naphthalene acts as an isothermal surface while the other surfaces are adiabatic. Even if the mass/heat transfer coefficient is obtained with an isothermal condition it can be applied to other boundary conditions, because the mass/heat transfer coefficient is a weak function of the wall temperature distribution in turbulent flows [1]. Furthermore, the nature of mass transfer allows one to impose these boundary conditions such that errors analogous to conductive and radiative losses in a wall are not present. The method thus readily distinguishes pure convection from conduction and radiation in conjugate heat transfer situations. The heat transfer coefficient, which is often desired, can be readily determined from the measured mass transfer results with good confidence via the mass/heat transfer analogy. Although the analogy requires that the Schmidt $(S c)$ and Prandtl $(\mathrm{Pr})$ numbers are of similar values, the naphthalene sublimation method is still applicable to many heat transfer problems under certain conditions. The Schmidt number for naphthalene vapor in air is 2.28 at $25^{\circ} \mathrm{C}$, which is sufficiently close to the Prandtl number of numerous gases and liquids to apply the analogy with good confidence. The method does not require any complex heating and integrated measuring system, such as insulating material and thermocouple junctions. The naphthalene sublimation technique needs a naphthalene-coated specimen and an accurate geometry or weight measurement system. The test specimen can be easily prepared by several methods, including dipping, machining, spraying, and casting. It is also possible to make a complex geometry including moving parts with the exact representations of the desired boundary conditions. The local transfer coefficients can be determined with high accuracy and in detail by automated measurement systems that eliminate most human errors. However, the naphthalene sublimation method cannot generally be used in certain flow situations, such as high-velocity flows due to aerodynamic and viscous heating (recovery effect) and natural convection due to the thermal buoyancy effects of the sublimation latent heat $[1,2]$. The naphthalene sublimation technique has been successfully used for the study of the mass and heat transfer in a variety of applications, which include external flows, flow in ducts and channels, heat exchanger, heat transfer enhancement, electronic cooling and rotating transfer surfaces [1-4].

The objective of the present work is to assess the naphthalene sublimation technique for characterization of the convective heat transfer in fundamental and industrial applications. The naphthalene technique has been used in a combination with modern surface scan methods to measure the naphthalene sublimation rate. Two configurations are used: jet impingement and an electric generator model. For the fundamental impinging jet case, the results from the naphthalene sublimation method are compared with other experimental results. This will hopefully show that the method is correctly used and can therefore, be translated to the electric generator case. The impinging jet case is performed for a jet exiting a long nozzle to ensure that the upstream flow field is fully developed. Heat transfer distributions produced by jets with nozzle-to-plate distances of 6 and 8 diameters are considered. Two Reynolds numbers, $R e$, of 15000 and 23000, based on the mean air flow velocity in the nozzle and the nozzle inner diameter are considered. For the industrial electric generator case the Reynolds number, $R e_{d h}$, is 4100 , based on the inlet hydraulic diameter of the ventilation ducts of the electric generator stator and the bulk velocity of the air entering those channels.

Impinging jets have become well-established as a highperformance technique for heating, cooling or drying surfaces. Applications of impinging jets include drying of textiles, film, and paper; annealing of glass; processing of some metals and glass; cooling of gas turbine components and the outer wall of combustors; cooling of electronic equipment; and freezing of tissue in cryosurgery. The wide area of application keeps the 
interest in impinging jets growing. The heat transfer rates associated with impinging gaseous jets have been well recognized and documented for many years [6-11, 13-16]. On the other hand, the evaluation of the naphthalene sublimation technique for studies of convective heat transfer in electric generator model aims to demonstrate the capability of this technique in real engineering problems. In such problems the other available techniques may not be feasible due to limitations in size or accessibility of the part under study, making the application of a heat source and/or the reading of temperatures extremely complicated. The importance of heat transfer and cooling of electric generators has been thoroughly discussed in the literature [12, 18-21]. Possible main issues with insufficient cooling of electric generators are reduced efficiency, hot-spots, and eventual failures. A better insight into the heat transfer phenomena for conventionally cooled generators will guide the design of these machines to avoid such problems.

The naphthalene sublimation technique has been used before for cases of fundamental and industrial applications as described in review papers $[1,2]$. However, no study has been found that uses the naphthalene sublimation technique for assessing the heat transfer in specific complex configurations such as electric generators, which are largely used in the industry. The goal of the present paper is therefore, to fill this gap. Moreover, the naphthalene sublimation technique is pushed in this study to new accuracies by using modern surface scanning methods (not used in older papers) and therefore, the heat transfer is resolved with as small sublimation as possible. This is important to avoid surface variations due to sublimation that can affect the flow pattern [1].

This investigation is divided into three main sections. First, the experimental methodology is described in detail, including the procedure for obtaining a good quality naphthalene specimen, which is critical for the success of the experiments. Then the methodology used for running the Computational Fluid Dynamics (CFD) simulations in the electric generator case is presented. In the final section, the results from the experiments and CFD simulations are presented and discussed.

\section{Experimental methodology}

The naphthalene sublimation technique uses the analogy between heat and mass transfer to determine convection coefficients. There are typically five steps to consider:

1. Provide a specimen with naphthalene.

2. Measure the initial naphthalene surface shape or the initial mass of the naphthalene specimen.

3. Conduct the experiment with the naphthalene specimen.
4. Measure the naphthalene surface shape or mass of the specimen after the experiment and obtain mass transfer rates from the sublimation depths or from the difference in mass together with the time the specimen was subjected to the flow.

5. Reduce the data to obtain the mass/heat transfer coefficient.

\subsection{Impinging jet setup}

The naphthalene specimens are in the present experiments made by casting. A mold is designed and manufactured to produce a $100 \mathrm{~mm} \times 100 \mathrm{~mm}$ naphthalene flat surface specimen. The mold is made of a $120 \mathrm{~mm} \times 120 \mathrm{~mm}$ highly polished aluminum plate with a filling hole and several air vents on the back for pouring melted naphthalene into the mold, see Fig. 1. The polished surfaces are cleaned before each casting, and a flat and smooth lid is screwed on top to complete the mold before the naphthalene is poured into it. The mold is heated to reach a constant favorable temperature when slowly filling up the mold, which is necessary to get a high-quality surface finish. The naphthalene is melted by heating a sufficient amount of naphthalene powder on an electric heater until it starts boiling. The boiling naphthalene is removed from the heater and is allowed to stop boiling. After a short time (about $30 \mathrm{~s}$ ) it is poured into the warm mold at a constant rate. The temperature and waiting time before pouring are important. A temperature that is too high results in small bubbles appearing on the naphthalene cast surface. A too low naphthalene temperature results in wavy traces on the test surface, formed during the filling. The air inside the mold can escape through the air vents to ensure that the entire mold is filled up. The excess of molten naphthalene that is poured into the mold comes out of the air vents and filling hole and sink back into the mold during the cooling process. The mold with the naphthalene is allowed to slowly cool down by free convection to room temperature during several hours. Finally, the lid is unscrewed and opened by gently sliding the lid. If the final result is successful, the smoothness of the exposed naphthalene surface is comparable to that of the polished metal adjacent to it. The surface of the specimen is inspected by eye and if it is flat it is ready for the test. Imperfections that are not visible to the eye are acceptable, since the surface must only be flat enough for the flow to experience a flat surface. The geometry of the surface is measured both before and after the test, so any imperfection will not influence the measurement of the sublimation.

The impinging jet setup is shown in Fig. 2. It consists of a long straight nozzle, a connection pipe to the compressed air supply system, a filter, a pressure regulator, a valve, a flow meter, a 1D traversing system, and a structure that holds the traversing system. The filter makes sure that the jet consists of 

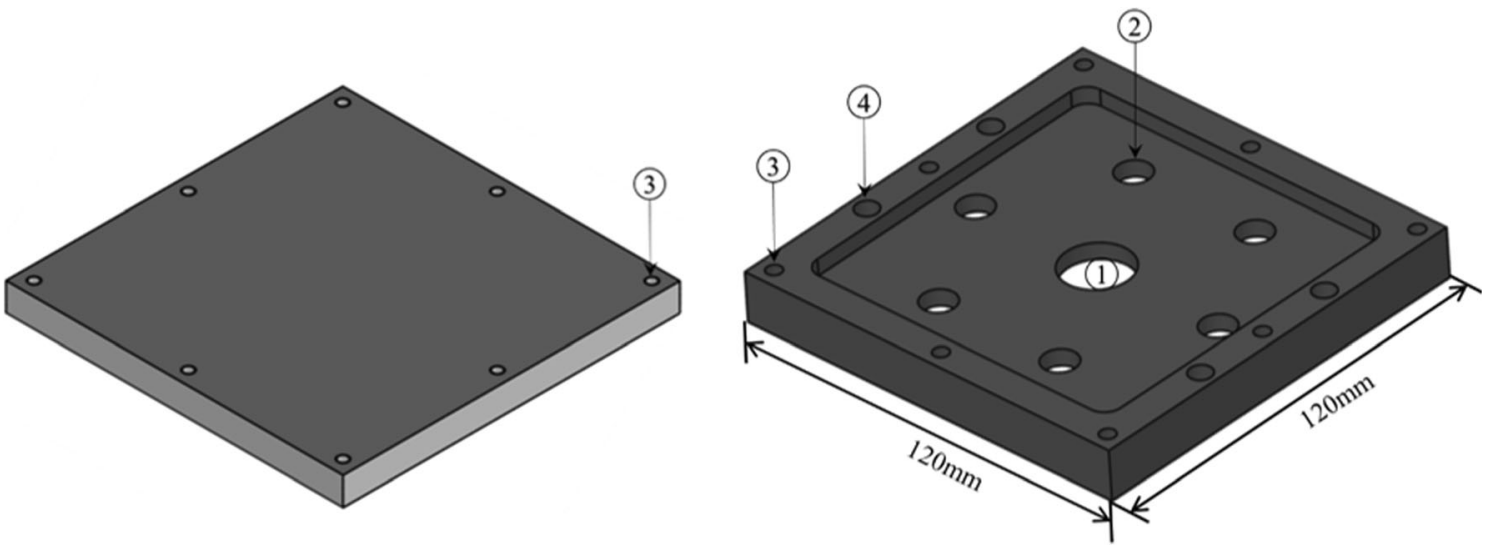

Fig. 1 Naphthalene specimen mold (right) and its lid (left). 1: Filling hole, 2: Air vents, 3: Lid fastener holes, 4: Holes to fix the specimen to the test setup

pure air. The pressure regulator and valve control the volume flow rate into the nozzle. A variable-area flow meter (rotameter) from Brooks Instrument, model 1307, with a tube of type R-9 M-25-3F and a RS float type is used. The flow meter full scale range is $21 \mathrm{~m}^{3} / \mathrm{h}$ and it can measure the flow rate with an accuracy of $\pm 2 \%$ and a repeatability of $\pm 0.5 \%$ of the full-scale range. A manually actuated linear translation stage with a micrometer step sensitivity is employed to traverse the nozzle in the direction normal to the naphthalene surface. It has been discussed by many authors that fully developed conditions can be reached in pipes with a length-todiameter ratio of around 50 and some authors even claim that this number can be lower [23-26]. The straight circular steel nozzle with a length-to-diameter ratio of 83 ensures a fully developed jet at the outlet of the nozzle. The jet temperature, compared to the ambient temperature, plays a very important role. Two Pt 100 resistance temperature detectors (RTD), connected on the outer surface of nozzle and covered with an insulation layer, are applied to get a good estimation of the air jet temperature. Another Pt 100 RTD is used to measure the ambient temperature.

The shape of the surface of the naphthalene specimen should be measured before and after the test in order to obtain

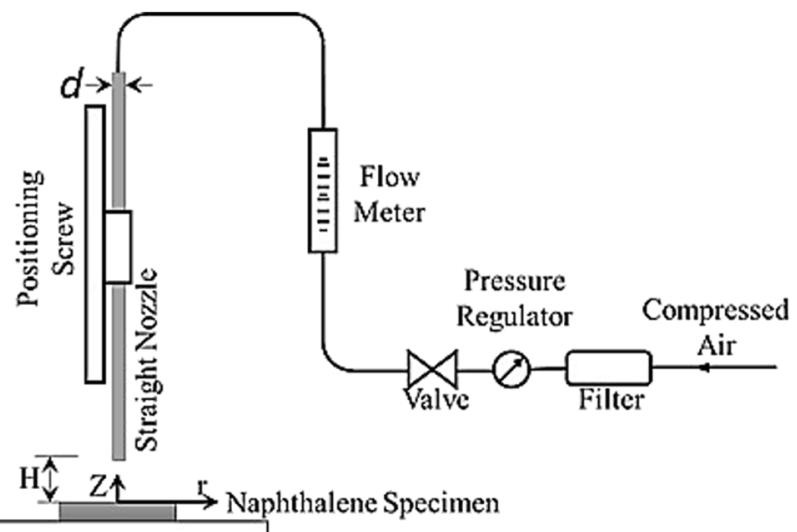

Fig. 2 Experimental setup. $H$ : Distance from nozzle exit to surface, $Z$ : Normal coordinate, $r$ : Radial coordinate, $d$ : Inner diameter of the nozzle the sublimation rate. These geometry measurements are done by a multi-sensor coordinate measuring machine (CMM) of the type VideoCheck® from WERTH INC. A touch trigger probe is used for the measurements, with a higher density of measurement points in the stagnation region and a lower density on the rest of the naphthalene surface. Overall 250 points are measured. The aluminum mold surface and corners are used as references during the measurements to make sure that the same points are measured before and after each test. The CMM was programmed to do the measurements automatically, to avoid human interaction and errors. The CMM is kept in a room with a controlled temperature of $20 \pm 1{ }^{\circ} \mathrm{C}$ and a humidity of $50 \pm 5 \%$.

The test time is set so that the naphthalene sublimation depth (surface elevation change during the test) is less than $200 \mu m$ in the normal direction. This ensures that the surface shape change has a minimum effect on the flow field, but at the same time the resolution is sufficient for an accurate measurement of the sublimation depth. In the case of $R e=15000$ the run time is $25 \mathrm{~min}$, while for $R e=23000$ it is $20 \mathrm{~min}$.

The sublimation process is active also when the specimen is not in the rig, i.e. during the geometry measurement, during storage and transportation, and during installation in the rig. The time the specimen is subjected to those natural convection events is recorded and used to correct the sublimation depth. The average natural convection sublimation rate is $1.3 \mu \mathrm{m} / \mathrm{h}$ in the current measurement conditions. This was determined by measuring the sublimation rate of a specimen exposed to natural convection for five hours. The sublimation depth at several points on the surface, and for each hour, was used in the averaging process.

\subsection{Electric generator setup}

Figure 3 shows the rig used for the electric generator experiments, which is the one described by Jamshidi [12]. This rig consists of a hydro-generator model with an inlet section, a rotor coupled to an electric motor, a stator and an external fan 
Fig. 3 The generator model. The big red oval insert to the left shows a zoom of the top and bottom pieces that are removable in all the four rows of the stator. The red rectangular insert shows one of the bottom pieces of those removable stator rows, including its channels. The top piece provides a flat surface on top of the bottom piece

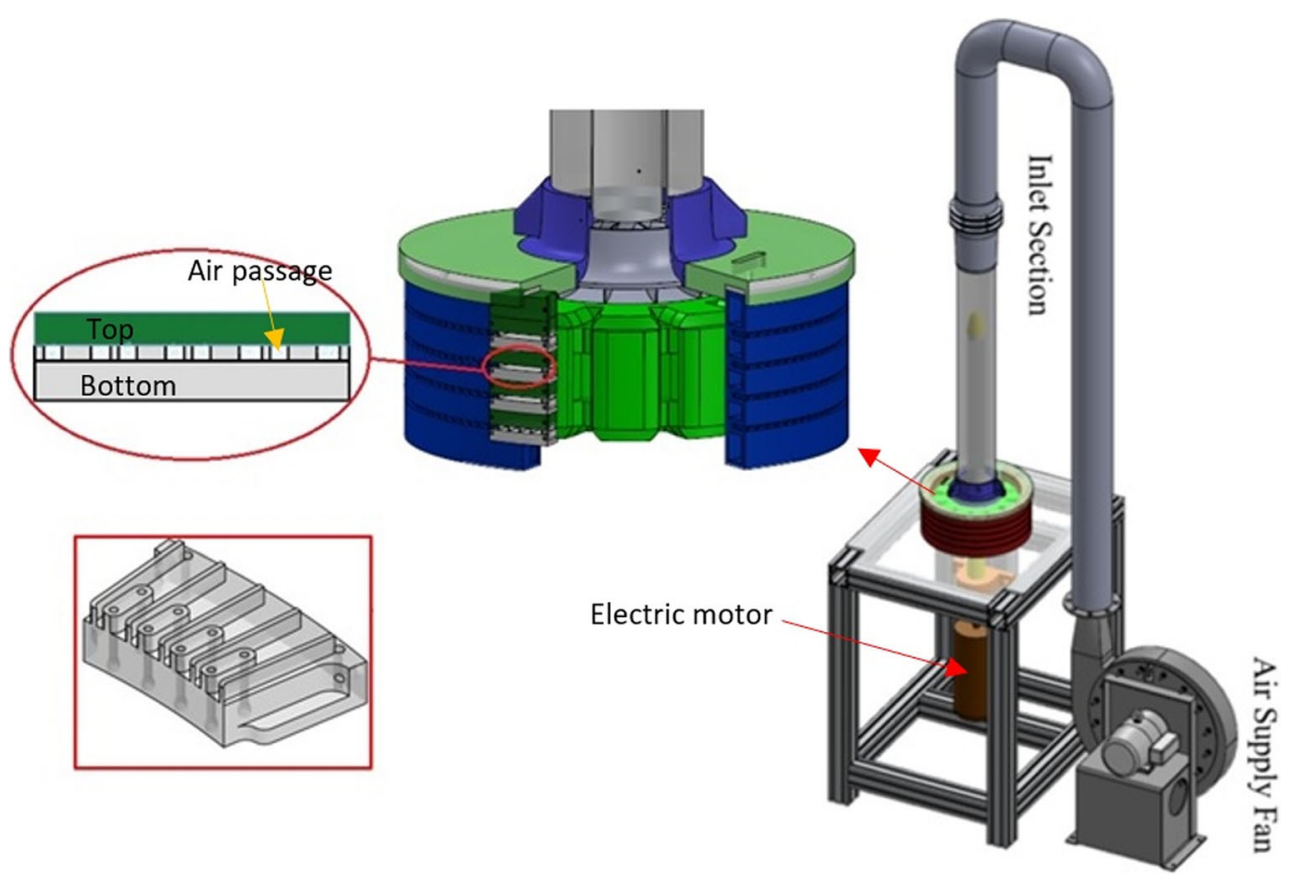

gas path, as seen in Fig. 4b. This makes sure that the top and bottom pieces can be firmly pressed together without disrupting the naphthalene surface. Four molds were manufactured to speed up the experiments, although they were used one at a time.

The naphthalene sublimation rate is in this case evaluated in two different ways, by weighting and scanning. However, neither the generator rig nor the CMM used for measuring the local sublimation rate for the impinging jet could be moved (they are located $40 \mathrm{~km}$ apart), making it impossible to use the same scanning equipment as for the impinging jet. Due to the strong smell of the naphthalene, it was not allowed to use any of the scanning equipment available at closer distance. Further, the most available scanning equipment at close distance was based on laser, which was shown not to be appropriate for the partly transparent naphthalene surface. The scanning thus involves an intermediate step where imprints of the specimen are taken using a dentistry elastomeric impression silicone, see Fig. 5. This material is used primarily for reproducing with high fidelity the shape of teeth, so high-quality reproducibility of the specimen shape is assured. These imprints are taken from all the specimens before and after the tests. Internal features of the mold are defined as references to be located with high accuracy before and after the tests, to be used to align the data during analysis. A laser scanner MikroCAD Lite is used to measure the shape of the imprints. The measurement area of the scanner is $26 \times 20 \mathrm{~mm}^{2}$, the lateral resolution is $16 \mu \mathrm{m}$ and vertical resolution of $1.6 \mu \mathrm{m}$. The noise of the measurement (short wavelength spikes) are reduced by using a Gauss filter with a cut-off of $0.08 \mathrm{~mm}$. Three areas of each ventilation channel are scanned from the imprints, shown in Fig. 5 with different colors. The blue procedure as discussed in section 2.1 . The result is a surface consisting of naphthalene only on the sidewall of the 


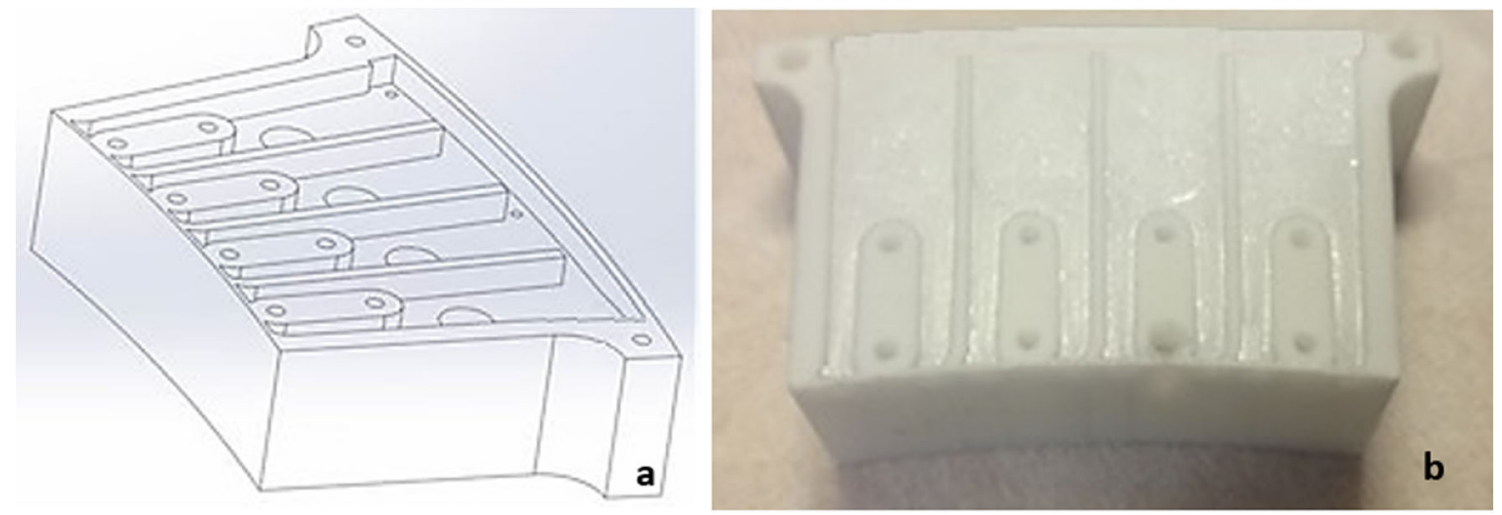

Fig. 4 a) CAD drawing of the mold of the top piece. b) Resulting specimen with flat naphthalene top channel surfaces, and supports for coils and spacers

squares show the wake regions, located behind the coils. The yellow rectangles show the downstream sides of the coils (DS), and the red rectangles show the upstream sides of the coils (US). The global sublimation rate is measured by weighting the naphthalene specimen before and after the experiments with a Sartorius BP 211D scale with weighting capacity up to $210 \mathrm{~g}$ and resolution of $10 \mu \mathrm{g}$, operating at an average temperature of $27 \pm 1{ }^{\circ} \mathrm{C}$.

The naphthalene specimen is mounted into the generator model once the initial imprints are taken and the specimen is weighted. The fan is then switched on. Tests were previously carried out to find a suitable exposure time of the specimen to the air flow. As previously described for the impinging jet experiment, the change of the shape of the naphthalene should have a minimum effect on the flow field, but at the same time the resolution should be sufficient for an accurate measurement of the sublimation depth. The required exposure time was set so that the surface elevation change is at least $20 \mu \mathrm{m}$. After $25 \mathrm{~min}$ of operation the fan is switched off, the naphthalene specimen is removed, and imprints and weights are taken. The differences in the weights and shape of surfaces before and after the test are used to find the sublimation rate.

\subsection{Data reduction for the impinging jet}

The local mass transfer coefficient $h_{m}$, and later the Sherwood number $S h$, are obtained from the local sublimation depth $\delta Z$ and the duration of the experiment $\delta t$. The local mass transfer coefficient $h_{m}$ is defined as

$h_{m}=\frac{\dot{M}^{\prime \prime}}{\rho_{v, w}-\rho_{v, \infty}}=\frac{\rho_{s} \delta Z / \delta t}{\rho_{v, w}}$

where $\dot{M}^{\prime \prime}$ is the local naphthalene mass flux. Since the fresh air introduced into the jet contains no naphthalene vapor, the naphthalene density of the upstream air is zero $\left(\rho_{v, \infty}=0\right)$ in this experiment. The naphthalene vapor density, $\rho_{v, w}$ is calculated from the perfect gas law and the naphthalene vapor pressure has been derived from the work of Ambrose et al. [5]. $\rho_{s}$ is the density of the solid naphthalene. The Sherwood number, based on the radius $r$, can be defined as

$S h=\frac{h_{m} r}{D_{\text {naph }}}$

The naphthalene vapor diffusivity in air, $D_{\text {naph }}$, is calculated from the correlation given by Goldstein and Cho [2].

$D_{\text {naph }}=0.0681\left(\frac{T}{298.1}\right)^{1.93}\left(\frac{1.013 \times 10^{5}}{P_{\text {atm }}}\right) \times 10^{-4}$

where $D_{\text {naph }}$ is in $\mathrm{m}^{2} / \mathrm{s}, T$ is in Kelvin and $P_{\text {atm }}$ in $\mathrm{N} / \mathrm{m}^{2}$. Finally, the Nusselt number can be calculated from the Sherwood number via the analogy
Fig. 5 Imprints taken from the naphthalene specimen with the four ventilation channels. The blue squares show the wake region. The yellow and red rectangles show the DS and US, respectively

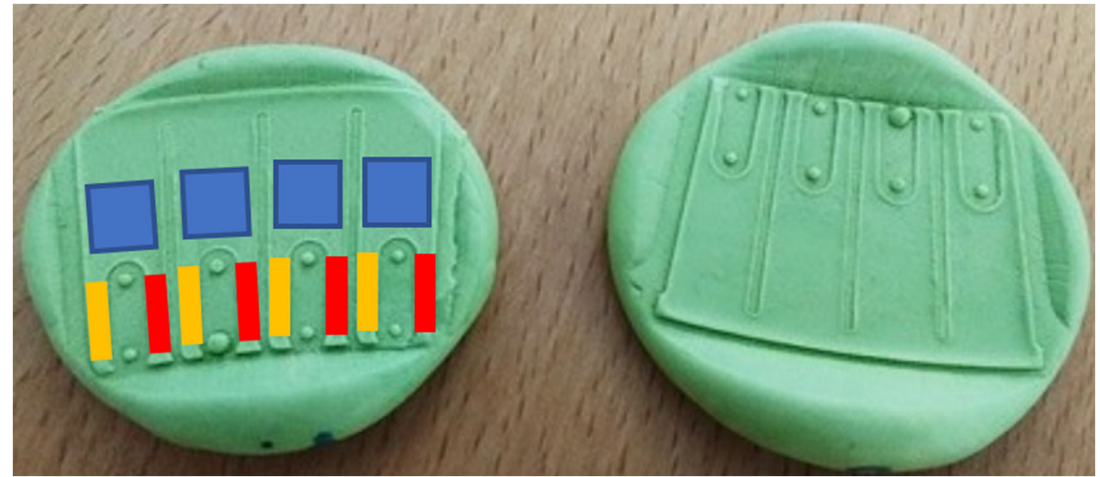


$\frac{N u}{S h}=\left(\frac{\mathrm{Pr}}{S c}\right)^{n}$

where $\operatorname{Pr}$ and $S c$ are the Prandtl and Schmidt numbers, respectively. The exponent, $n$, is a constant determined from empirical results and has been found to be in the range of $1 / 3$ to 0.4 . In the impinging jet case $n=0.36$ is used as suggested in [6].

\subsection{Data reduction for the electric generator}

Two sources of data are available: from scanning the imprints and from weighting the specimens. The data reduction from the scanning follows a similar procedure as explained in section 2.3 for the impinging jet experiment. However, in this case the average heat transfer is determined in each of the measurement areas shown in Fig. 5, since it is difficult to obtain the local sublimation rate correctly in this complex configuration. The average of each area (US, DS and wake region) is based on all the four channels included in the imprint of each row. The total average values of $\overline{h_{m}}, \overline{S h}$ and $\overline{N u}$ for each row are then determined by an average of the three scanned areas.

The weighting of the specimen yields the area-averaged mass transfer coefficient as

$\overline{h_{m}}=\frac{\delta m / \delta t}{A_{s}\left(\rho_{v, w}-\rho_{v, \infty}\right)}$

where the overall mass sublimation rate from the naphthalene surface with area $A_{s}$ is determined from the difference in weights, $\delta m$, and the time duration of the test, $\delta t$. The mean Sherwood number is in this case defined in terms of the hydraulic diameter, $d_{h}$, of the ventilation channel, as

$\overline{S h}=\frac{\overline{h_{m}} d_{h}}{D_{\text {naph }}}$

The diffusivity and $\overline{N u}$ are calculated from Eqs. (3) and (4). In Eq. (4) the value of $n=0.4$ for internal flows is used [1, 22].

\subsection{Uncertainty estimates}

Uncertainty estimates for the measurements in this investigation have been made from the accuracy of measurement devices and by repeatability tests. For the impinging jet case, the accuracy of the CMM is $\pm 1.5 \mu \mathrm{m}$. For the electric generator case, the standard error of the scanning procedure is determined as $\pm 6.9 \mu \mathrm{m}$ and for the weighting procedure, the accuracy is $\pm 0.2 \mathrm{mg}$. For the impinging jet case, the resulting uncertainty ranges from $\pm 0.35 \%$ at the highest $N u$ value to $\pm 1.5 \%$ at the lowest $N u$. For the electric generator case using the weighting method, the uncertainty for $\overline{N u}$ is estimated as \pm 0.5 . If the scanning procedure method is used in the electric generator case, then the uncertainty for $\overline{N u}$ is \pm 5 .

\section{Numerical procedure}

CFD simulations of the generator model configuration are performed as a complement to the experimental studies. The finite volume method is used in the FOAM-extend-3.1 CFD tool [27] to solve the thermal fields and the Reynolds-Averaged NavierStokes (RANS) equations for the flow field. The domain used for the electrical generator configuration is discretized in ICEM CFD. The numerical procedure is here presented.

The geometry used for the electric generator simulation is based on the one used by Jamshidi [12], shown in Fig. 6. Jamshidi used a 1/12th sector of the generator in the tangential direction in the rotor and intake zone, a 1/108th sector of the stator and the outlet region, and a mixing plane to couple those regions. This comprises one rotor pole and one fan blade passage, and one ventilation channel in each of the four channel rows. The part of the domain and mesh that is used in the present study is enclosed by dashed rectangles, including only the stator and the outlet region. The inlet is located where the mixing plane was located in the simulations by Jamshidi. This restriction in the computational domain is done in order to save computational time. The computational domain consists of a block-structured mesh with a total of $4.1 \mathrm{M}$ cells. The cell centers of the first layer of cells at the walls have $y^{+}$values below 1. Figure 6 also defines the upstream (US) and downstream (DS) sides of the ventilation channels, used in the analysis of the results.

The inlet boundary condition is a non-uniform velocity distribution. The values of the velocity components and the turbulence quantities are extracted from the results of Jamshidi [12], for the case of a flow rate of $0.16 \mathrm{~m}^{3} / \mathrm{s}$. Given that a mixing plane was used by Jamshidi at that location, both the cylindrical velocity components and the turbulence quantities have one-dimensional distributions along the axial extent of the stator inlet. The no-slip condition is imposed at all the walls. The inlet temperature is set to $300 \mathrm{~K}$ and the air properties are defined at this temperature. A constant heat rate is prescribed at the top walls of the ventilation channels, and during the simulation the temperatures of these wall are calculated by the solver. The remaining walls are assumed to be adiabatic. The homogeneous Neumann condition is used for all variables at the outlet, and cyclic boundary conditions are imposed at each side of the computational domain.

The SST $k-\omega$ turbulence model is used to complete the RANS equations. An accurate heat transfer can be calculated using the SST formulation as discussed in [28]. In addition, this model is particularly suitable because it can be used to integrate the boundary layer all the way to the wall and it has 

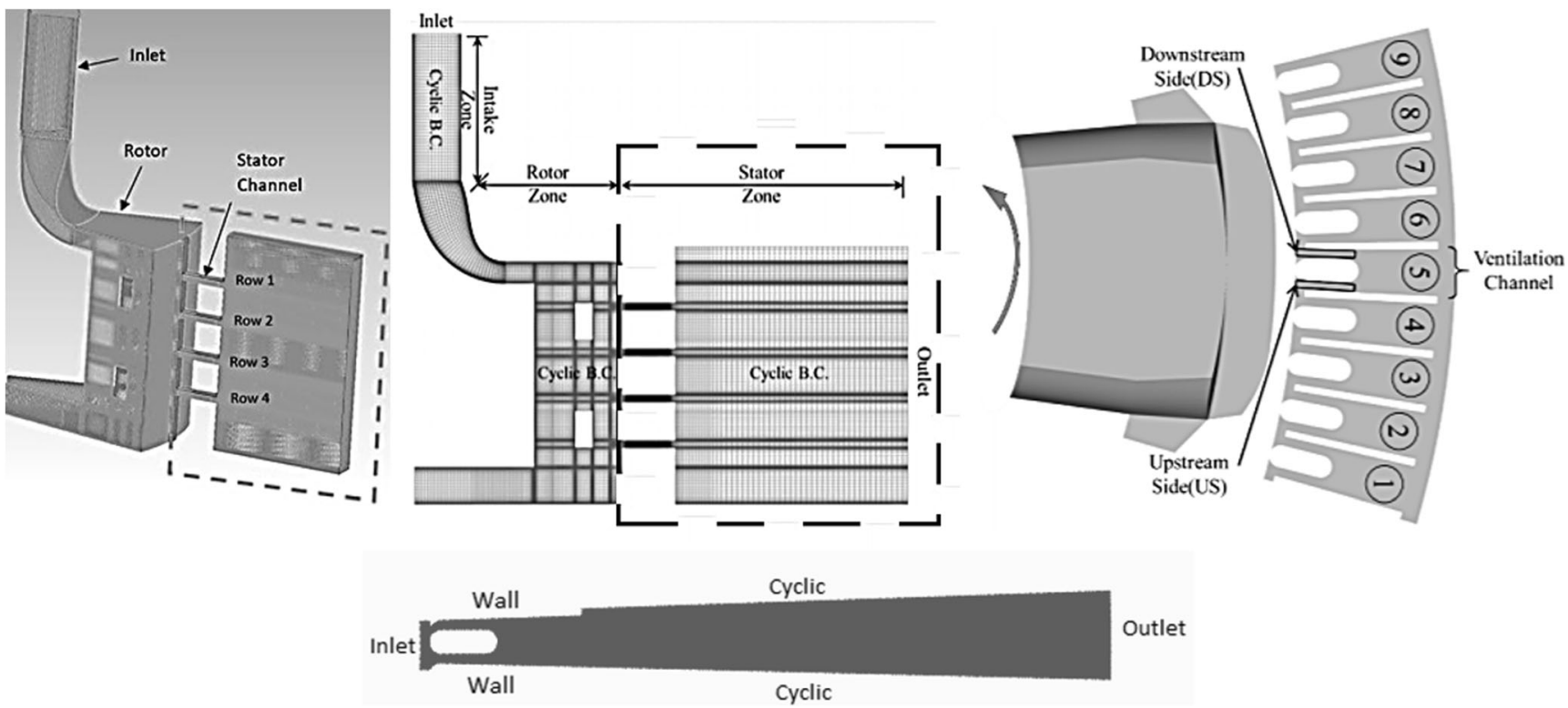

Fig. 6 Left: Domain used by Jamshidi [12]. Center: lateral view of the domain used by Jamshidi [12]. The dashed rectangles enclose the part of the domain used in the present study. Right: cross-section of the stator to show the upstream (US) and downstream (DS) sides of the ventilation

been found to be the best performing RANS model in terms of stability for this particular case. The SIMPLE algorithm is the numerical procedure that is used for solving the momentum equations and the Limited Linear numerical scheme is used for discretizing the convective term in the momentum equations. The Limited Linear numerical scheme is a second order numerical scheme that limits towards first order in regions of rapidly changing gradients [29]. The convergence of the simulations is monitored by checking the temperature of the fluid at selected locations and the residuals. Convergence is reached when the temperatures at the selected locations do not change, and when the residuals of the momentum and energy equations are reduced by at least three orders of magnitude.

\section{Results and discussions}

The results obtained from the two configurations are shown and discussed separately.

\subsection{Impinging jet results}

Figure 7 shows contour plots of the resulting sublimation depth along the naphthalene surface for $R e=23000$ and 15000 for $H / d$ ratios of 6 and 8 . The sublimation is not perfectly axisymmetric, probably due to surface imperfections. The sublimation depths on the specimen are therefore circumferentially averaged, yielding radial distributions from the center of the jet impingement. channels (only one channel is included in the simulations, but nine channels are shown here for clarity). Bottom: slice along the row 1 showing the boundaries

Figure 8 shows the Sherwood number on the surface of the naphthalene specimen for all the cases. The maximum value is at the stagnation point. In the vicinity of the stagnation region the Sherwood number reduces rapidly and then the change becomes more gradual in the wall jet region. A comparison of left and right plots in Fig. 8 shows that the Sherwood number at the same Reynolds number reduces if the jet distance to the surface increases. Consequently, the heat transfer should be reduced by increasing the $H / d$ ratio, for a given Reynolds number.

Figure 9 shows a comparison between the naphthalene sublimation technique results and results from experiments done by others. The Nusselt number $(N u)$ reduced from the sublimation data is accurate and comparable to the other experimental results. The agreement with the data from Lytle and Webb [13] is very good and the largest difference (around $20 \%$ ) is seen with the results from Yan [17]. The experimental results from the present study shows a similar trend as the other experimental data. A peak of the heat transfer is observed at the stagnation region and it reduces rapidly as $r / d$ increases with similar variation of $\mathrm{Nu}$ with $r / d$ (similar slope). The fact that the results from the naphthalene sublimation technique in the impinging jet case are accurate and comparable to others, show that the method has been used properly.

\subsection{Electric generator results}

Figure 10 shows the topologies resulting from the laser scans of the imprints taken from the first row, representing all the experiments. The surface enclosed in the blue dashed square shows the area that has been used as reference for levelling, 
Fig. 7 Sublimation depth from the impinging jet experimental cases: (a) $R e=23000$ and $H / d=6$, (b) $R e=23000$ and $H / d=8$, (c) $R e=15000$ and $H / d=6$, (d) $R e=$ 15000 and $H / d=8$
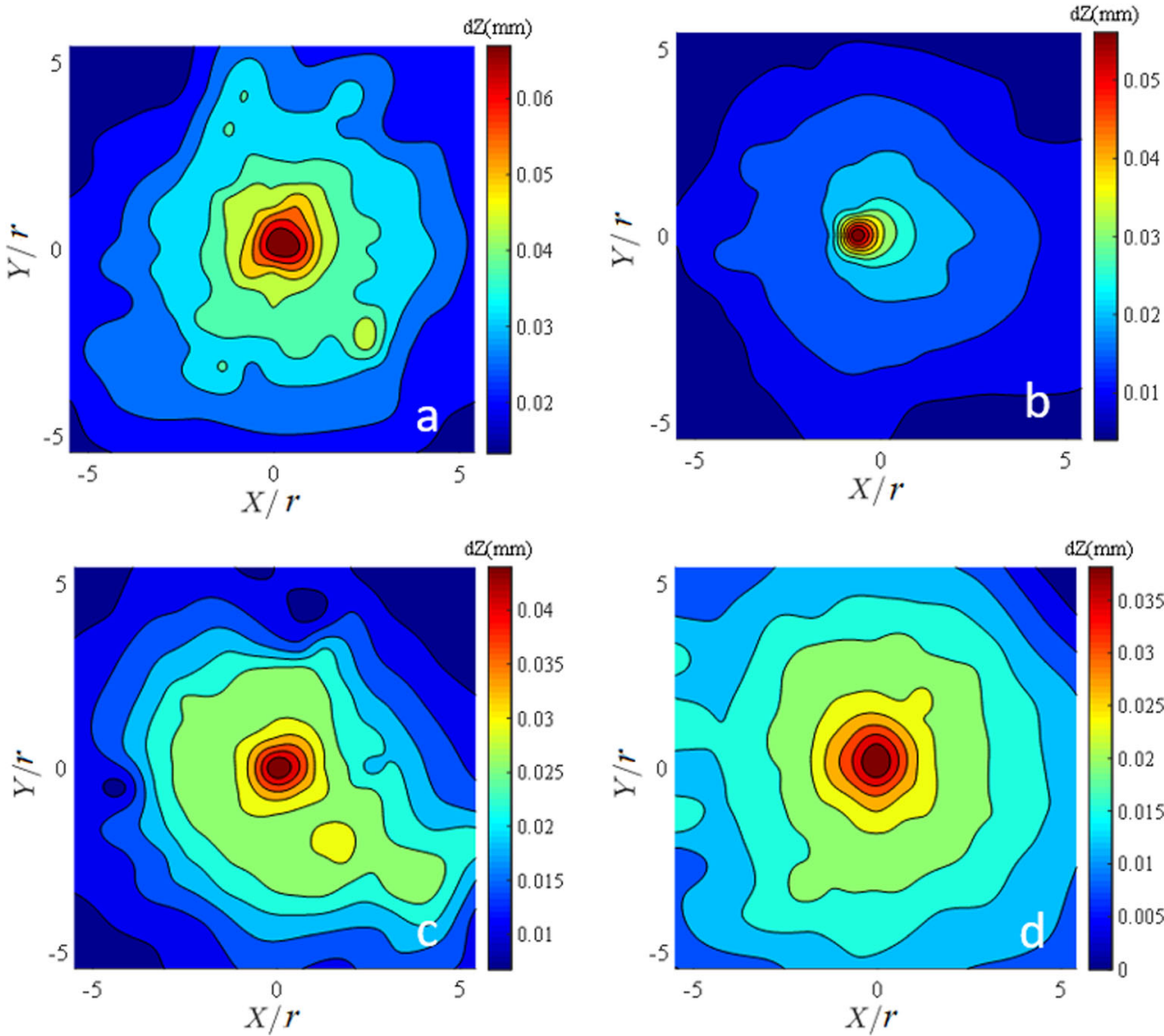

which is where the zero (0) elevation has been set. From the topology determined before the experiment, it can be seen that although precautions were taken during molding, the surface deviates slightly from being flat. The naphthalene surface is generally slightly more elevated than the mold surface, except for some slight depressions at the entrance of the channel. This imperfection is of the order of $100 \mu \mathrm{m}$. It is here assumed that this imperfection does not influence the flow, and since the method is based on the difference in topology before and after the experiment the results should be accurate. The topology after the experiment clearly shows that the depressions due to naphthalene sublimation are now accentuated at the entrance of both the DS and US, which means that the local sublimation rate is larger in this area. It is also shown that the naphthalene surface along the DS and US is less elevated than the reference surface, indicating also high sublimation rates. However, in the wake region the color of the surface is more similar to that of the reference surface, which means that less sublimation has occurred in the wake region.

More details of what occurs in the different rows can be seen in Fig. 11, showing height contours of the silicon imprints from the naphthalene surfaces in all the rows of the generator model after being scanned. The left and right images show the surfaces before and after the experiment,
Fig. 8 Sherwood number obtained from the sublimation depth of the impinging jet cases for both $R e=15000$ and 23000 . Left: for $H / d=6$. Right: for $H / d=$
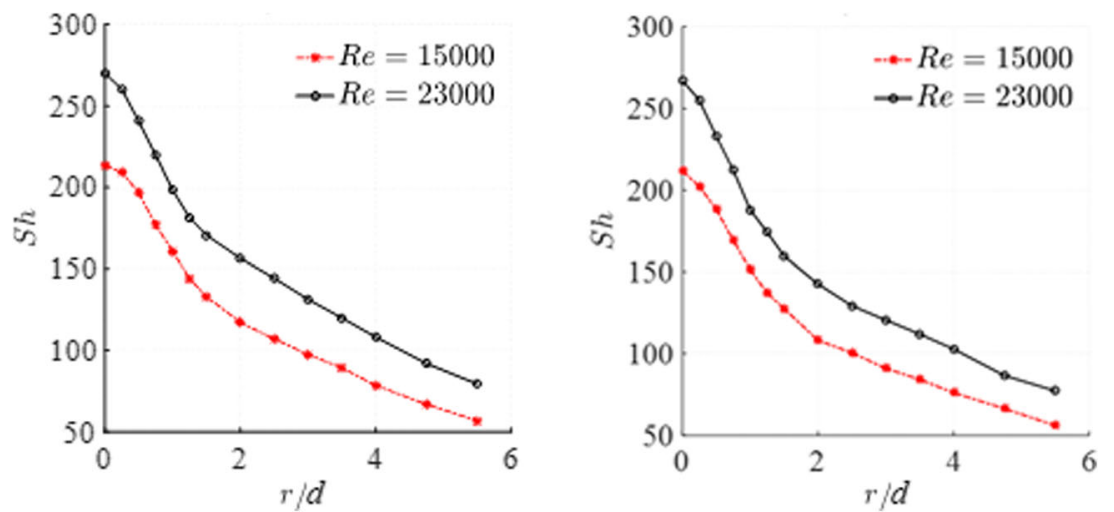
Fig. 9 Comparison of naphthalene sublimation technique results with results from impinging jet experiments by other authors for $R e=23000$ and $H / d=6$

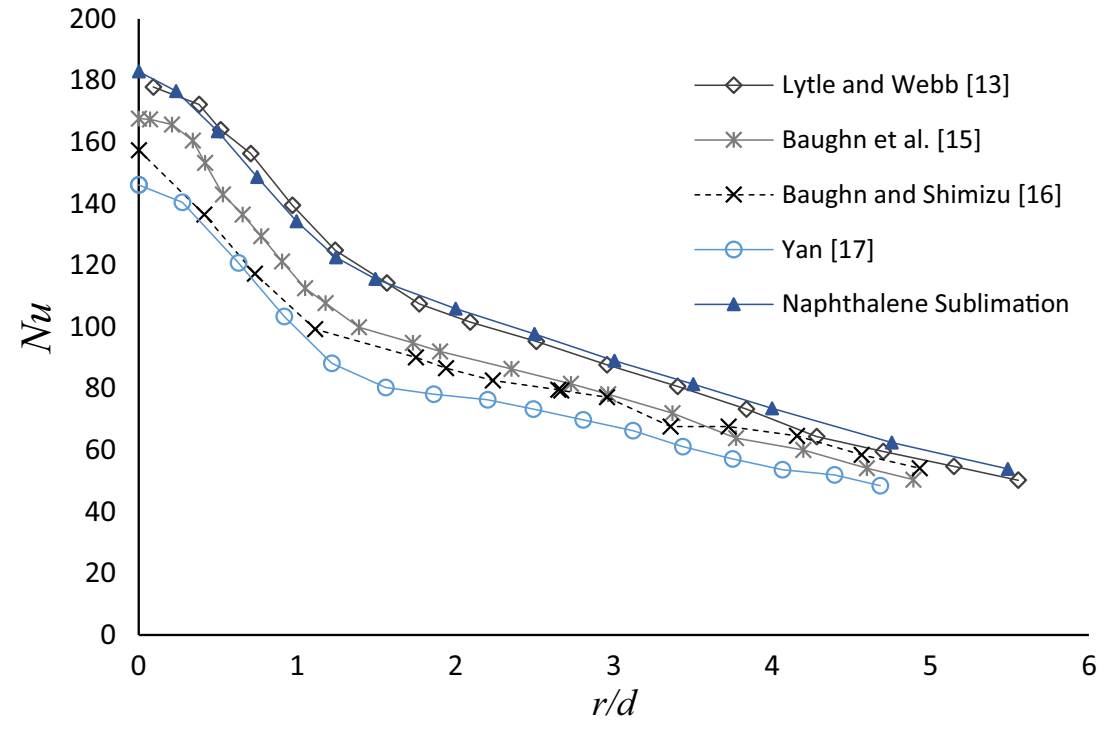

respectively. The zero-level surface in all the rows is located as illustrated in Fig. 10. It is shown that all the rows had slight depressions at the entrance of the DS and US ducts before the experiments and that in the wake region the naphthalene surface is generally in all rows more elevated than the reference surface. These imperfections indicate that in the future the naphthalene surface after the molding should be better finished/machined to get closer to a flat surface. It is also seen that the mold itself is not perfectly flat either. The elevations of the reference surface, for example, tend to be lower in the center of the surface. This is also observed in other mold surfaces. The imperfections that are caught in all the surfaces during the scanning indicate that the silicon imprints are a good choice for capturing features or details in any surface.

Regarding the local naphthalene sublimation, observed by comparing the images of the naphthalene surfaces before and after the experiment, it is seen from Fig. 11 that the sublimation rate is in general very large at the entrance of both DS and US ducts. However, as discussed above, this can be an overprediction caused by the imperfections already present before the experiments. The highest sublimation rates are seen at the downstream walls of both ducts in all the rows and tend to increase from row 1 to row 4 . The sublimation rate is lower at the upstream walls of the rows and tend to increase from row 1 to row 4 . The variation in sublimation rates that are seen in the different rows and ducts might be related to the fact that the flow distribution in general tends to be imbalanced in the ducts. However, the effect of the curvature at the inlet of the rows is beneficial for rows 3 and 4 and helps the flow being more balanced, as previously observed by Jamshidi [12]. The wake region also reveals differences in the rows. The sublimation rate is higher and similar in rows 3 and 4 compared to

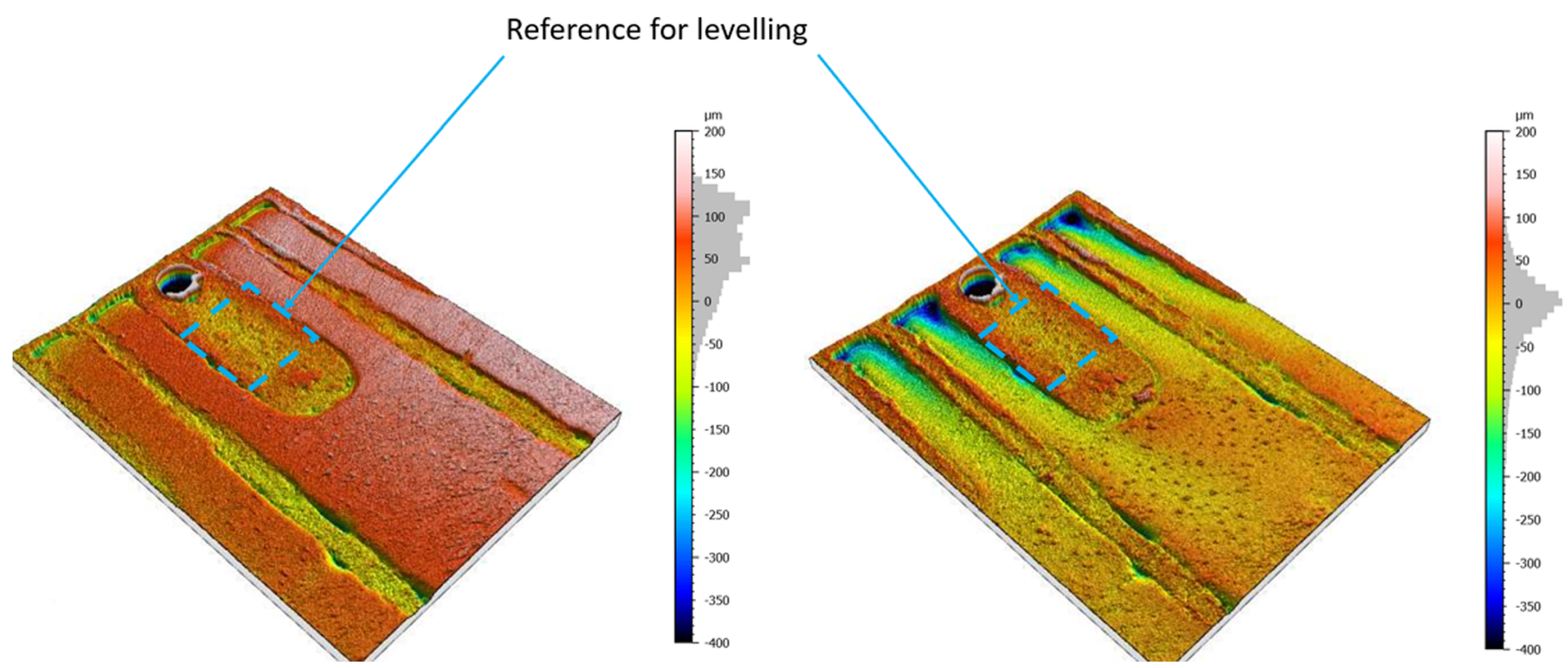

Fig. 10 Topology of the imprints taken from the specimen for the first row. Left: before the experiment. Right: after the experiment 
Fig. 11 Topologies of the naphthalene surfaces for rows $1-$ 4, from top to bottom for $R e_{d h}=$ 4100. Left images show surfaces before the experiments and the right images show surfaces after the experiments
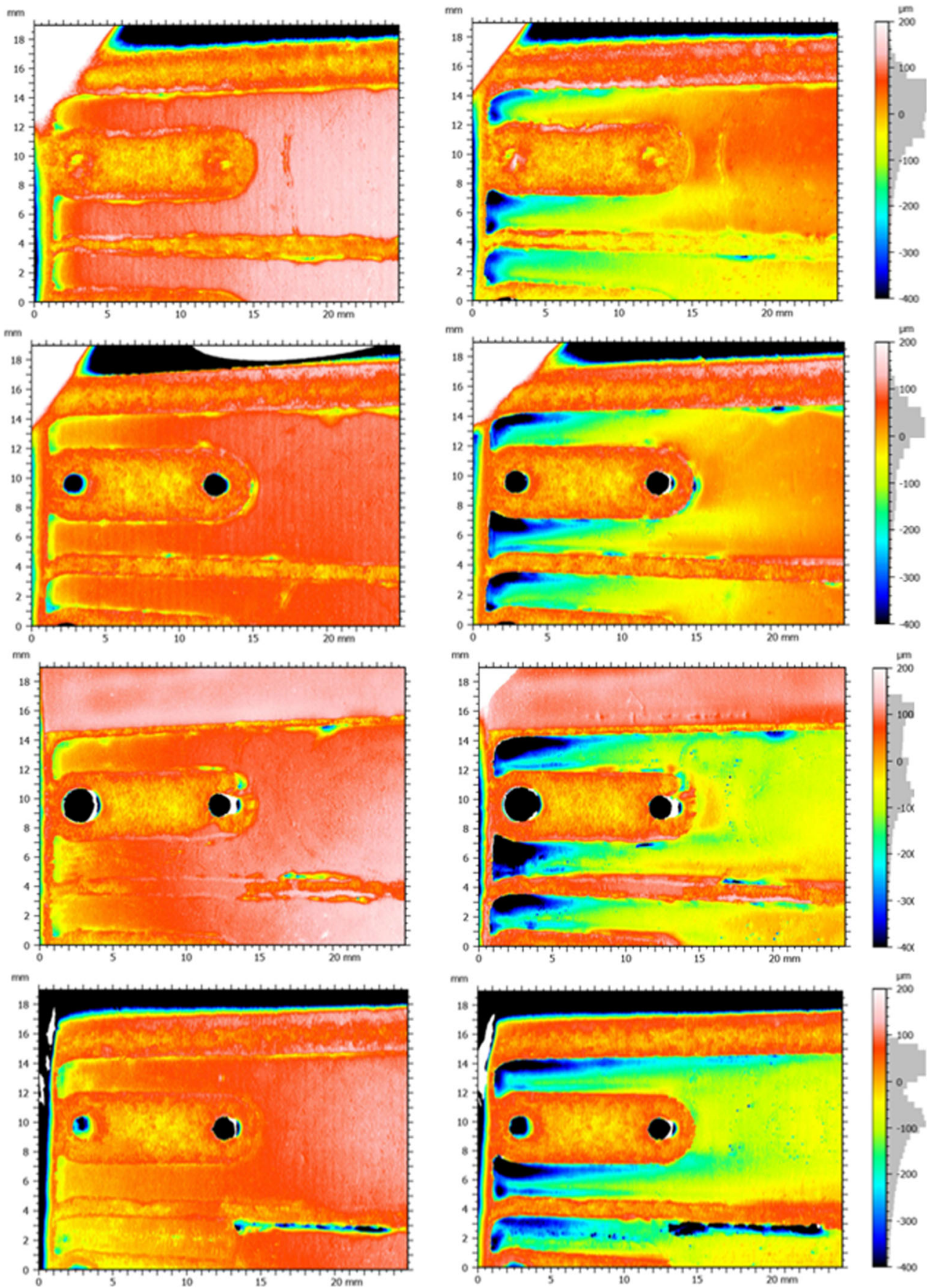

rows 1 and 2 . The imbalance in the ducts is also seen in Fig. 12, which shows the velocity contour from the CFD simulation of the generator model in row 1 . It is clearly seen that the flow experiences rapid acceleration and deflection as it enters the DS and US ducts, which could be the cause of the local increased sublimation rate that can be observed in all the rows. As shown by Fig. 11, the naphthalene surface was already locally depressed in this area before the experiments. Thus, there will as well be some additional effects due to the unavoidable mold wall that causes a small backward facing step at the entrance to the channel. The sublimation rate is therefore probably slightly over-estimated in this region. Figure 12 also shows that the flow velocity is high at the downstream walls of both ducts. The upstream walls of both ducts and the wake region are areas in which the flow velocity is reduced, and this might be related to the low sublimation rate that is observed in these regions, as previously mentioned.

The qualitative analysis of $\overline{N u}$ in the electrical generator model is considered in the present study to be more important than the quantitative determination of this 

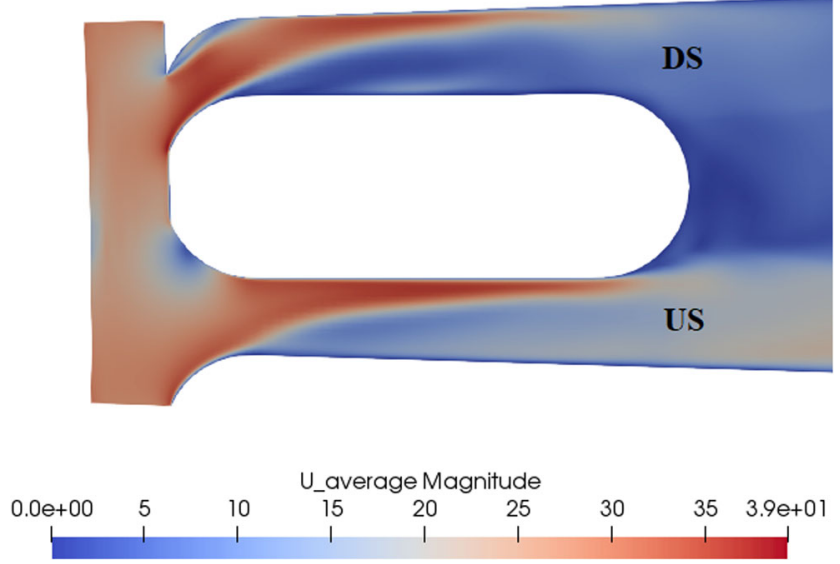

Fig. 12 Velocity contour plot from the CFD simulations of the generator model

parameter. Spotting relations among the convective heat transfer in the different rows of the stator and from the different methods is also found to be easier if $\overline{N u}$ is normalized as shown in Fig. 13. Here, the normalized $\overline{N u}$ obtained from both the experiments with weighting and scanning, and from the CFD simulation are presented. The normalized Nusselt number for each row, $\overline{N u_{N i}}$, is obtained from the ratio of the average Nusselt number determined in each row, $\overline{N u_{i}}$, and the value of the average Nusselt number in row 4 obtained by scanning, $\overline{N u_{4 s}}$. Thus,

$\overline{N u_{N i}}=\frac{\overline{N u_{i}}}{\overline{N u_{4 s}}}$

It is observed that the heat transfer is in general larger in rows 3 and 4 of the stator, which means better cooling in rows 3 and 4 compared with rows 1 and 2 . The results from the scanning and the results from CFD show that the heat transfer in row 2 is larger than in row 1 . The results from weighting say the opposite, but in any case, the difference is very small. The heat transfer is slightly larger in row 4 than in row 3 according to the results from weighting and from CFD. In this case the results from scanning say the opposite, but the difference in heat transfer between these two rows is also very small. The CFD results show a more even heat transfer among the different channel rows than the experimental results. The results from experiments show a $20 \%$ increase of the heat transfer from row 2 to row 3. The CFD results also show an increase, but to a smaller extent. The results from the weighting and CFD are generally closer than the results from the scanning and CFD, except for row 2 . The results from the scanning is always higher than the other two results and the difference is more evident in rows 3 and 4 , indicating a sensitivity of the experimental results to the way the sublimation depth is measured. Jamshidi [12] showed that there are larger tangential velocities and less axial velocities at the entrance of rows 3 and 4, providing better conditions for the pick-up shape (curvature at the inlet of the stator ducts) of the spacers and thus contributing to a larger mass flow through these channels. This can cause an increase of the convective heat transfer in those rows. The difference in the values between the two experimental methods is about $17 \%$, with a larger difference in the third row. If the uncertainty of the scanning procedure is taken into consideration, the difference in the normalized $\overline{N u}$ between both experimental methods could be reduced to $\sim 10 \%$. However, considering this article as a first step in using the naphthalene sublimation method for the determination of the heat transfer in this type of applications, further work in this field would be expected to improve the experimental accuracy. When comparing the weighting results of the experiments with the CFD results, an average of 5\% in difference is obtained (CFD results higher). The difference between results from the scanning and CFD results is in average $13 \%$. In any case, the CFD results are lower.

Figure 14 shows the normalized $\overline{N u}$ at the downstream side of the coils (DS), from both the experiment with scanning and from CFD. The normalization was also calculated as shown
Fig. 13 Normalized $\overline{N u}$ for the different rows from the CFD simulation of the generator model and from the experiments using weights and scanning to determine the naphthalene sublimation rate

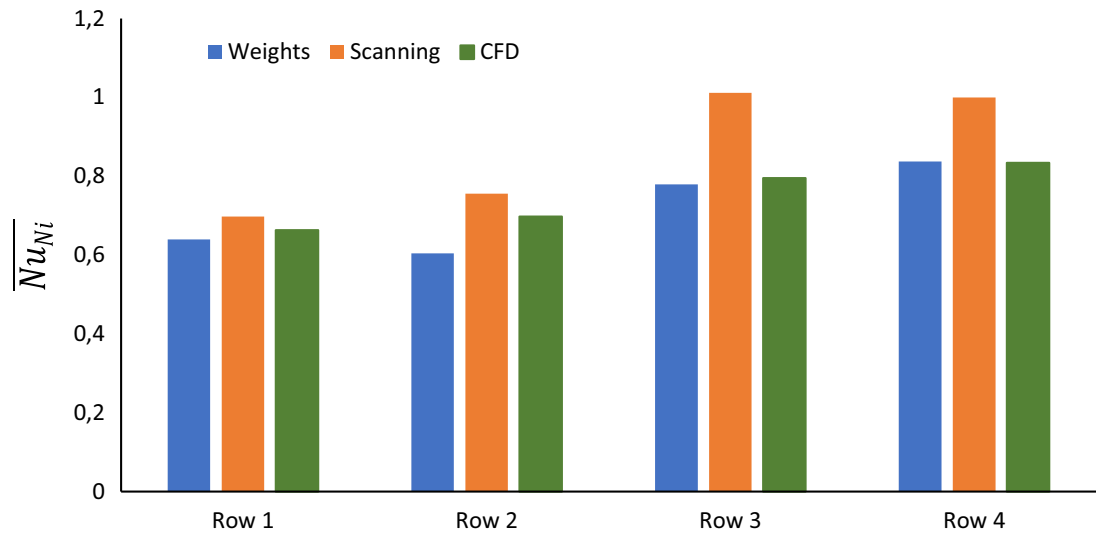


Fig. 14 Normalized $\overline{N u}$ of the DS of the coils for the different rows of the generator model

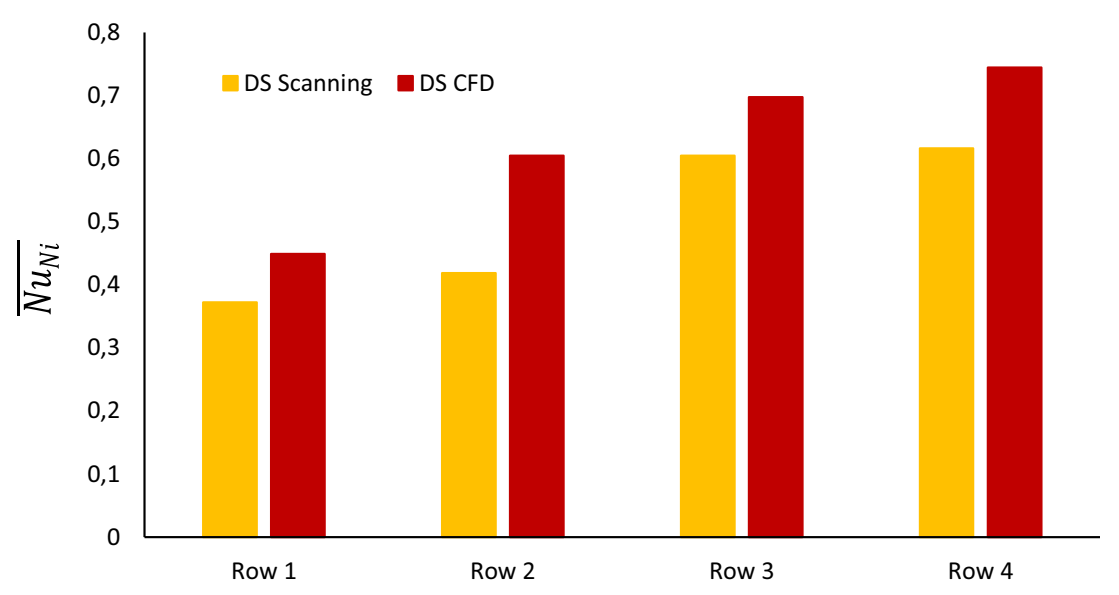

by Eq. (7), but in this case $\overline{N u_{i}}$ is the average Nusselt number in only the DS. The experimental and numerical results agree on the fact that the heat transfer increases at the DS from rows 1 to 4 . The experimental data shows that the heat transfer does not change much from row 1 to row 2 and from row 3 to row 4 , but changes by $25 \%$ from row 2 to row 4 . The CFD results show that the heat transfer change occurs gradually from row 1 to row 4. The CFD results are larger in all the rows, with the largest difference with the experiments in row 2 . The average difference between experiments and CFD results is $\sim 20 \%$.

Figure 15 shows similar data as in Fig. 14 but for the US. Here the CFD results are also higher than the experimental results. The CFD results show a gradual increase of the heat transfer from row 1 to row 4 . For the experiments, the difference in heat transfer is more visible from row 2 to row 3. For both CFD and experimental results the heat transfer is larger in rows 1 and 2 , compared to what is observed for the DS. At the same time, the heat transfer is in general slightly reduced in the US for rows 3 and 4 compared to the DS, according to the experimental results. For row 4, the heat transfer is slightly larger than the heat transfer that was obtained for the DS according to CFD. From what can be observed for rows 1 and 2, it is safe to conclude that the US in rows 1 and 2 perform better than the DS in row 1 and 2 . The best agreement between the experimental and numerical results is seen in row 3 and the largest difference is seen in row 2 . The average difference between $\mathrm{CFD}$ and experimental results is $\sim 22 \%$.

In general, the semi-local values of the heat transfer for the DS and US for the experiments using scanning of the naphthalene surface are lower than the CFD results. However, as seen in Fig. 13, the heat transfer from the scanning is always higher than the CFD results and the weighting results. This might indicate that the wake region affects in greater way the heat transfer of the scanning results compared to what can be seen from the CFD and the weights. This could be further investigated in the future with a different scanning procedure that is able to measure all the wake region behind the coil. This would give a more realistic estimation of the heat transfer in the wake region and more realistic contribution of this region to the overall heat transfer of the ventilation channel.
Fig. 15 Normalized $\overline{N u}$ of the US of the coil for the different rows of the generator model

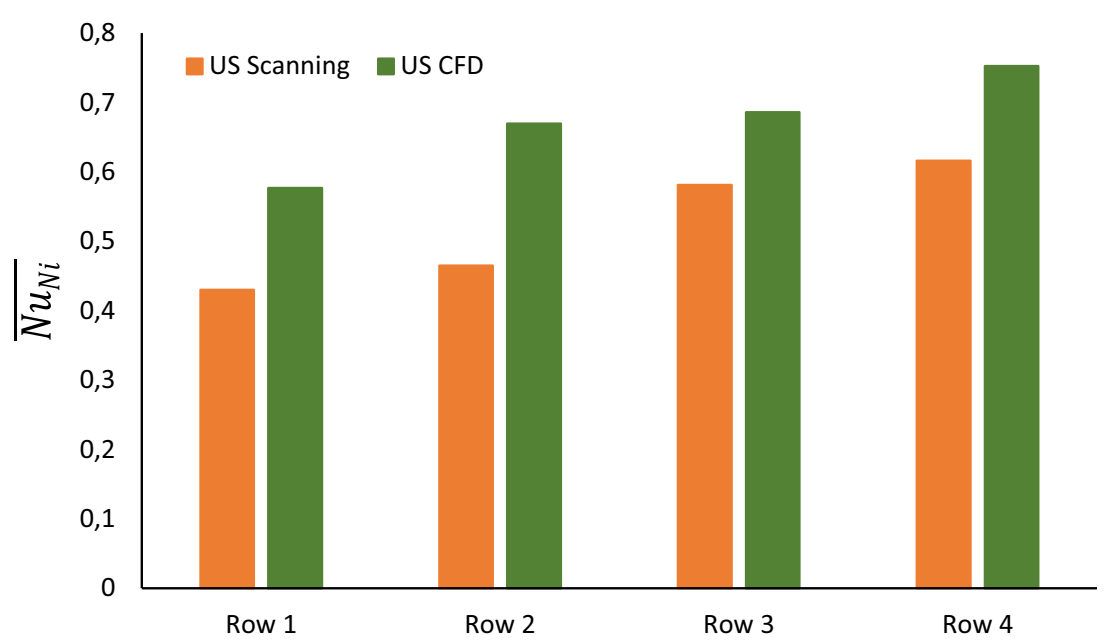




\section{Conclusions}

1. An assessment of the naphthalene sublimation technique for characterization of the convective heat transfer in fundamental and industrial applications has been carried out and the results compared with already published experimental data and CFD simulations. Two configurations were used: jet impingement and an electric generator model.

2. For the electric generator model, the naphthalene sublimation rate was obtained by measuring the difference in weights of the naphthalene specimens before and after the experiments, and by measuring the sublimation depth before and after the experiments. For the case of the impinging jet only the measuring of the sublimation depth is used for obtaining the heat transfer. For the impinging jet four different cases were considered, varying the nozzle distance to the impingement surface and the jet Reynolds number. For the case of the electric generator model, a single case was considered at a fully turbulent regime.

3. The results show that the naphthalene sublimation technique is in general, capable of capturing the heat transfer characteristics in cases of both fundamental and applied research. The heat transfer in the jet impingement was closely predicted to what other studies have shown, and for the electric generator case, the results from the naphthalene sublimation technique is capable of showing areas in the stator channels where the heat transfer is poor and could ideally be enhanced. This is important for improving the cooling capabilities of these machines, which results in improved efficiency during their operation. In the generator model case, the flow behavior and distribution in the rows have a big impact on the heat transfer, and in general the results from CFD and experiments were congruent with what has been found in the past for the same configuration.

4. It has been feasible to determine the heat transfer in cases of fundamental and industrial applications with as small naphthalene sublimation as possible. Modern scanners can measure very small variations in the shape of the naphthalene surface for an accurate determination of the sublimation rate.

5. The preparation of the specimen with naphthalene is an important factor for the success of the experiments, and some directions regarding how to obtain a good specimen with a naphthalene surface of high quality were given. It was also discussed that not only the molding procedure is important, but the manufacturing of the mold itself implies some considerations that are important to take into account to ensure that the results are reliable.

6. The silicone imprinting captured with faithfulness all the features and imperfections not only of the naphthalene surfaces, but also those present in the mold. The imprinting of naphthalene surfaces is useful in cases in which the scanning equipment is not close-by and/or when laser-based scanning is used as it was the case in the present study.

7. The measurement technique in the experiments has a big impact on the results, with differences close to an average of $17 \%$ between the results using scanning and weighting. The selection of the measurement technique depends on the available equipment for measurement, but in general more studies would eventually determine which technique give the most accurate results.

8. In the electric generator case, the results of the overall heat transfer from the naphthalene sublimation technique were closer to the CFD results. The weighting method of measuring the sublimation rate was closer to the CFD results with average difference of $5 \%$. The scanning results differed from CFD with about $13 \%$. For the semi-local assessment of the heat transfer in both DS and US ducts, the difference between experiments and CFD was larger. The wake region might be influencing in larger extend the experimental results from the scanning, compared to what can be seen from the CFD results and the weighting method.

Acknowledgements Open access funding provided by Chalmers University of Technology. The research presented was carried out as a part of the "Swedish Hydropower Centre - SVC". SVC has been established by the Swedish Energy Agency, Energiforsk and Svenska Kraftnät together with Luleå University of Technology, The Royal Institute of Technology, Chalmers University of Technology and Uppsala University. www.svc.nu. The simulations were performed on resources provided by the Swedish National Infrastructure for Computing (SNIC) at C3SE and NSC.

\section{Compliance with ethical standards}

Disclosure statement There is no conflict of interest identified and/or reported by the authors.

Open Access This article is licensed under a Creative Commons Attribution 4.0 International License, which permits use, sharing, adaptation, distribution and reproduction in any medium or format, as long as you give appropriate credit to the original author(s) and the source, provide a link to the Creative Commons licence, and indicate if changes were made. The images or other third party material in this article are included in the article's Creative Commons licence, unless indicated otherwise in a credit line to the material. If material is not included in the article's 
Creative Commons licence and your intended use is not permitted by statutory regulation or exceeds the permitted use, you will need to obtain permission directly from the copyright holder. To view a copy of this licence, visit http://creativecommons.org/licenses/by/4.0/.

\section{References}

1. Souza Mendes PR (1991) The napthalene sublimation technique. Exp Thermal Fluid Sci 4(5):510-523

2. Goldstein RJ, Cho HH (1995) A review of mass transfer measurements using naphthalene sublimation. Exp Thermal Fluid Sci 10(4): 416-434

3. Kwon HG, Hwang SD, Cho H (2011) Measurement of local mass/ heat transfer coefficients on a dimple using naphthalene sublimation. Int J Heat Mass Transf 54(5):1071-1080

4. Park J, Yoo S (2004) A naphthalene sublimation study on mass/heat transfer for flow over a flat plate. KSME international journal 18(7): $1258-1266$

5. Ambrose D, Lawrenson IJ, Sprake CHS (1975) The vapour pressure of naphthalene. J Chem Thermodyn 7:1173-1176

6. Livingood J. N. B and Hrycak P. "Impingement heat transfer from turbulent air jets to flat plates - a literature survey", NASA Technical Memorandum (NASA TM X-2778), 1970

7. Martin H (1977) Heat and mass transfer between impinging gas jets and solid surfaces. Adv Heat Transfer 13:1-60

8. Jambunathan K, Lai E, Moss MA, Button BL (1992) A review of heat transfer data for single circular jet impingement. Int $\mathrm{J}$ Heat Fluid Flow 13:106-115

9. Viskanta R (1993) Heat transfer to impinging isothermal gas and flame jets. Exp Thermal Fluid Sci 6:111-134

10. Gardon R, Cobonpue J (1962) Heat transfer between a flat plate and jets of air impinging on it. ASME, Int. Develop. Heat Transfer, pp 454-460

11. Hrycak P (1983) Heat transfer from round impinging jets to a flat plate. Int J Heat Mass Transf 26:1857-1865

12. Jamshidi H (2017) Ventilation flow field characteristics of a hydrogenerator model - an experimental and numerical study. Chalmers University of Technology, Dissertation

13. Lytle D, Webb BW (1994) Air jet impingement heat transfer at low nozzle plate spacings. Int J Heat Mass Transf 37:1687-1697
14. Behnia M, Parneix S, Durbin P (1996) Simulation of jet impingement heat transfer with the k-e-v2-f model. Center Turbulence Research Annual Research Briefs:3-16

15. Baughn JW, Hechanova AE, Yan X (1991) An experimental study of entrainment effects on the heat transfer from a flat surface to a heated circular impinging jet. J Heat Transf 113(4):1023-1025

16. Baughn JW, Shimizu S (1989) Heat transfer measurements from a surface with uniform heat flux and an impinging jet. J Heat Transf 111(4):1096-1098

17. Yan X (1995) A preheated-wall transient method using liquid crystals for the measurement of heat transfer on external surfaces and in ducts. Previews of Heat and Mass Transfer 5(21):459-460

18. Moradnia P, Golubev M, Chernoray V, Nilsson H (2014) Flow of cooling air in an electric generator model - an experimental and numerical study. Appl Energy 114:644-653

19. Moradnia P, Chernoray V, Nilsson H (2014) Experimental assessment of a fully predictive CFD approach, for flow of cooling air in an electric generator. Appl Energy 124:223-230

20. Jamshidi H, Nilsson H, Chernoray V (2015) CFD-based design and analysis of the ventilation of an electric generator model, validated with experiments. Int J Fluid Mach and Syst 8:113-123

21. Toussaint K, Torriano F, Morissette J, Hudon C, Reggio M (2011) CFD analysis of ventilation flow for a scale model hydrogenator. ASME Power Conference 2:627-637

22. Sparrow EM, Ramsey JW (1978) Heat transfer and pressure drop for a staggered wall-attached array of cylinders with tip clearance. Int J Heat Mass Transf 21:1369-1377

23. Schlichting H (1966) Boundary-layer theory, Sixth edn. McGrawHill, New York

24. Panton R (2005) Incompressible flow. Wiley, Third Ed

25. Incropera F, Dewitt D, Bergman T, Lavine A (2013) Principles of heat and mass transfer. Wiley, Seventh Ed

26. Grenson P, Léon O, Reulet P, Aupoix B (2016) Investigation of an impinging heated jet for a small nozzle-to-plate distance and high Reynolds number: an extensive experimental approach. Int J Heat and Mass Transfer 102:801-815

27. Website foam-extend Open Source CFD Toolbox http://foamextend.org

28. Menter F R. "Two-equation eddy-viscosity turbulence models for engineering applications", AIAA J. 32, no. 8 (1994) 1598-1605

29. OpenFOAM User Guide version 6, July 2018. https://openfoam.org

Publisher's note Springer Nature remains neutral with regard to jurisdictional claims in published maps and institutional affiliations. 